FORMATION Formation emploi

Revue française de sciences sociales

141 | Janvier-Mars 2018

Quand le tutorat questionne le travail et son analyse

\title{
Le rôle des usagers dans la formation aux métiers de services à l'aune des interactions tutorales
}

The role of users in training for service professions in the context of tutorial interactions

Die Rolle von Benutzern in Ausbildungssituationen für Dienstleistungsberufe : Ein Blick auf die Interaktionen zwischen Auszubildenden und Tutoren El papel de los usuarios en la formación en las profesionales de servicios según las interacciones de tutoría

\section{Laurent Filliettaz}

\section{CpenEdition} Journals

Édition électronique

URL : http://journals.openedition.org/formationemploi/5345

DOI : 10.4000/formationemploi.5345

ISSN : 2107-0946

Éditeur

La Documentation française

Édition imprimée

Date de publication : 15 avril 2018

Pagination : 45-66

ISSN : 0759-6340

Référence électronique

Laurent Filliettaz, « Le rôle des usagers dans la formation aux métiers de services à l'aune des interactions tutorales », Formation emploi [En ligne], 141 | Janvier-Mars 2018, mis en ligne le 15 avril 2020, consulté le 30 octobre 2020. URL : http://journals.openedition.org/formationemploi/5345 ; DOI : https://doi.org/10.4000/formationemploi.5345 


\title{
Le rôle des usagers dans la formation aux métiers de services à l'aune des interactions tutorales
}

\begin{abstract}
LAURent FiLLiettaz
Professeur ordinaire à la Faculté de psychologie et des sciences de l'éducation de l'Université de Genève, dans le domaine "Formation des adultes, langage et travail ». Linguiste de formation, il dirige l'équipe Interaction \& Formation, au sein du Laboratoire Recherche, Intervention,
\end{abstract}

Formation et Travail (RIFT)

Résumé

Le rôle des usagers dans la formation aux métiers de services, à l'aune des interactions tutorales

Cet article vise à décrire de manière méthodique les effets que peuvent produire, sur les pratiques de formation, les situations de travail qui ne portent pas seulement sur des objets techniques et matériels, mais aussi sur des acteurs humains, des " usagers ", dans le cadre de relations dites de service. Nous mobilisons une analyse vidéo-ethnographique d'interactions tutorales se déroulant dans des institutions de la petite enfance et dans un centre de radiologie en milieu hospitalier. Les analyses produites révèlent que les usagers - respectivement des patients et des enfants - contribuent activement à faire évoluer les situations d'interaction et qu'ils agissent de manière significative sur les conditions de participation des stagiaires et des tuteurs.

Mots clés : métier de service, relation de service, stagiaire, tutorat, processus d'apprentissage, analyse du travail, enseignement technique-professionnel, formation en alternance, stage de formation, Suisse

Abstract

The role of users in training for service professions in the context of tutorial interactions

This article aims at describing in a methodical way how work situations involving the provision of services to " users " may entail specific contingencies and opportunities for novice workers when they have to learn practical skills during internships. The paper is based on video-ethnographic case studies, focusing on verbal and non-verbal interactions between trainees, workplace tutors and users in two distinct institutional contexts: early childhood education and a radiology center in a public hospital. The analyzes produced reveal that users - respectively patients and children - actively contribute to shape interac- 
tion processes and that they significantly affect the conditions through which trainees and tutors participate to work production tasks

Keywords: occupation in the service sector, service relationship, trainee, tutoring, learning process, work analysis, technical \& vocational education, sandwich training, traineeship, Switzerland

Journal of Economic Literature: M 53 ; M 54

Traduction : Auteur.

\section{Introduction : les usagers dans la modélisation des interactions tutorales}

De manière surprenante, la littérature récente portant sur les rapports entre langage, travail et formation n'a que peu abordé la question de la place des " usagers » dans la formation aux métiers de service. Certes, en sociologie et en linguistique du travail, d'abondants travaux empiriques soulignent l'importance croissante des métiers de service dans le contexte de la "nouvelle économie " et d'une société dite de l'information et de la connaissance (Borzeix \& Fraenkel, 2001 ; Boutet, 2001, 2008 ; Joseph, 1995), mais sans pour autant s'intéresser aux enjeux de formation.

La didactique professionnelle et l'ergonomie de tradition francophone n'ont pas manqué de s'intéresser aux professions dites de "service " et plus généralement à "l'analyse d'activités qui s'accomplissent avec d'autres humains " ${ }^{1}$. Selon Pastré, Mayen $\&$ Vergnaud (2006), les activités de services présentent souvent une complexité accrue par rapport aux activités productives observables en contexte industriel : a) elles requièrent un haut degré de conceptualisation, b) elles présentent une grande diversité et variabilité interne, c) l'accès au résultat de l'action n'est souvent pas direct et accessible, $\mathrm{d}$ ) les effets produits ne dépendent pas toujours du seul agent, e) les activités diagnostiques y sont omniprésentes et portent sur des dimensions différentes du travail, f) elles confèrent à la fois une grande autonomie à l'action et l'obligation de respecter des procédures explicites, g) enfin, elles génèrent souvent de la souffrance chez des travailleurs, liée notamment à l'impossibilité de répondre aux demandes.

La didactique professionnelle et l'ergonomie de tradition francophone ont clairement intégré les métiers dits de service dans le périmètre de leur domaine de recherche et d'intervention. Pourtant, force est de constater que les interactions de tutelle constituent un objet d'investigation dans lequel la " figure de l'usager " a encore été peu explorée. En effet, depuis quelques années, on assiste à un regain d'intérêt de la recherche en

1. Cf. Cerf \& Falzon, 2005 ; Hubault, 2002 ; Pastré, 2011 ; Pastré, Mayen \& Vergnaud, 2006. 
formation professionnelle pour l'activité des tuteurs et les interactions de tutelle ${ }^{2}$. Ces travaux ont permis de cerner quelques-unes des spécificités des activités de tutelle, et en premier lieu leur caractère organisé et distinct des pratiques d'enseignement scolaire (Savoyant, 1995 ; Boccara et al., 2015).

Pourtant, le périmètre empirique de ces travaux reste encore très majoritairement dominé par les professions techniques, artisanales et agricoles. En outre, et surtout, les " modélisations " proposées de l'activité tutorale reposent sur une vision fortement dyadique de la relation entre "novices" et "experts». Les pratiques d'étayage décrites par Mayen (2002) et par Chrétien (2014) dans le domaine agricole, ou par Kunégel (2011) dans le domaine de la mécanique automobile, permettent de repérer des formats à la fois récurrents et dynamiques de la relation tutorale en situation de travail. Mais cette relation reste conceptualisée comme un rapport binaire entre deux agents. On néglige ici de considérer que cette relation porte sur des agents qui sont eux-mêmes des partenaires de l'interaction.

Dans ce contexte, et dans le prolongement de nos travaux antérieurs portant sur les interactions tutorales en formation professionnelle initiale ${ }^{3}$, cet article s'intéresse à l'étude de situations de formation dans et par le travail, dans lesquelles le travail ne consiste pas seulement en une transformation matérielle du monde extérieur, mais en l'établissement d'une relation avec des " usagers ».

La présence des usagers dans le périmètre du travail constitue une contingence particulière qui ne manque pas d'exercer des influences notables sur l'activité. Elle requiert un " travail relationnel " et interactionnel avec l'usager, en parallèle aux enjeux de formation qui caractérisent classiquement la relation tutorale elle-même (Rémery \& Markaki, 2016). À ce titre, elles procèdent de ce que l'on pourrait qualifier de relation " au carré", une relation portant sur une relation (Filliettaz, op. cit.). Mais la présence des usagers dans le périmètre du travail peut constituer aussi une opportunité pour la formation, dès lors qu'elle agit sur les situations, et plus spécifiquement sur les conditions dans lesquelles les tuteurs médiatisent la rencontre des novices avec les réalités du travail.

C'est ce que notre démarche souhaite explorer, en décrivant, de manière méthodique, les effets que peuvent produire, sur les pratiques de formation, les situations de travail qui ne portent pas seulement sur des objets techniques et matériels, mais aussi sur des acteurs humains, des " usagers ", dans des relations dites de service.

Pour ce faire, deux corpus empiriques présentant des similarités dans leurs conditions de recueil seront examinés. Ils portent respectivement sur deux formes contrastées de

2. Cf. Balslev et al., 2015 ; Billett, 2001 ; Fuller \& Unwin, 2003 ; Mikkonen et al., 2017.

3. Voir notamment Filliettaz, de Saint-Georges \& Duc, 2008 ; Filliettaz, Rémery \& Trébert, 2014 ; Filliettaz, 2015 ; Filliettaz \& Rémery, 2015 ; Filliettaz \& Trébert, 2016 ; Filliettaz \& Durand, 2016. 
relations de service. D'une part, la relation de soin qui s'établit entre des techniciens en radiologie médicale et leurs patients. D'autre part, la relation éducative qui s'opère entre des éducateurs et des enfants fréquentant des institutions de la petite enfance. À partir d'une documentation vidéo-ethographique d'interactions tutorales se déroulant, dans ces contextes, entre des stagiaires, des tuteurs et des usagers, les analyses proposées auront pour objectif de questionner les effets formatifs des interventions des usagers dans les interactions tutorales.

La première partie de l'article explicite le cadre théorique de la démarche adoptée, ainsi que les conditions méthodologiques dans lesquelles la recherche a été menée. La deuxième partie propose deux études de cas, issues respectivement de chacun des deux corpus empiriques considérés. À l'aide d'extraits de transcriptions, elle montre comment les interventions des usagers, respectivement des patients et des enfants, peuvent exercer une influence sur le potentiel d'apprentissage des situations de travail rencontrées par les stagiaires. Enfin, la dernière partie explicite les conséquences d'un changement de regard sur le rôle des usagers dans les interactions tutorales. Elle formule, en outre, des propositions visant à concevoir des formes d'aménagement des situations de travail à même de soutenir ce potentiel d'apprentissage.

\section{Une perspective interactionnelle}

La démarche de recherche adoptée ici s'inscrit dans une perspective interactionnelle en analyse du travail (Filliettaz, 2014 ; Filliettaz, de Saint-Georges \& Duc, op. cit.). Si la didactique professionnelle peut être définie comme "l'analyse du travail pour la formation " (Pastré, op. cit.), la perspective interactionnelle contribue à préciser les conditions dans lesquelles l'analyse du travail peut être réalisée, notamment dans ses dimensions langagières, communicationnelles et plus généralement interactionnelles. C'est là une composante à la fois constitutive et significative de bon nombre d'activités de travail comme d'expériences de formation.

Adopter le point de vue de l'interaction sur les activités de travail et de formation, c'est considérer que les processus interactionnels constituent, pour les chercheurs comme pour les praticiens, tout à la fois des objets et des méthodes d'analyse. Ce sont d'abord des objets car ils forment un domaine de réalité qui médiatise l'accomplissement des activités de travail et de formation. Et ce sont des méthodes dans la mesure où ils peuvent être considérés comme des moyens par lesquels les acteurs comme les chercheurs produisent une compréhension locale de ces activités dans les conditions de leur accomplissement. De ce point de vue, les interactions tutorales ne sont pas appréhendées comme un processus abstrait et décontextualisé, mais comme un "accomplis- 
sement pratique " rendu manifeste par les conditions dans lesquelles il est réalisé par les participants (Filliettaz, 2010, 2014).

Sur le plan théorique, adopter un point de vue interactionnel sur les pratiques professionnelles accompagnées revient à assumer trois postulats. Le premier reconnaît la relation constitutive qui s'établit entre les comportements observables des membres d'un groupe et les conditions à la fois pratiques, matérielles, historiques et sociales dans lesquels ils sont réalisés et interprétés. En continuité avec une perspective d'inspiration ethnométhodologique (Garfinkel, 2007), ces comportements n'acquièrent leur caractère ordonné et signifiant que dans les conditions dans lesquelles ils sont accomplis. Ils sont pour ainsi dire contingents des circonstances dans lesquelles ils sont observables. Le deuxième postulat reconnaît le caractère conjointement construit et co-élaboré des réalités impliquées dans les interactions. Dans une perspective inspirée de l'analyse conversationnelle (Sacks, 1992), la signification et l'ordre des actions situées s'établissent conjointement, étape par étape, et dans un processus permanent de négociation entre les partenaires de l'interaction. Cette signification n'est pas donnée a priori. Elle n'est pas non plus le fait d'actions isolées et appréhendées individuellement. Elle résulte d'une logique d'enchaînement séquentiel et d'interdépendances entre les tours de parole (Schegloff, 2007 ; Traverso, 2016). Enfin, le troisième postulat reconnaît le caractère à la fois langagier et plus généralement multimodal des processus par lesquels se co-construit et se négocie la signification en acte des comportements des participants aux interactions. Dans la lignée des approches multimodales en analyse du discours et de l'interaction (Kress et al., 2001 ; Goodwin, 2000 ; Mondada, 2004), ces significations sont fondées, d'une part, sur une large palette de ressources issues de systèmes sémiotiques variés (la parole, la prosodie, les gestes, les postures corporelles, les objets matériels, les pratiques scripturales etc.) et, d'autre part, sur des combinaisons et des formes d'articulation entre ces ressources.

En cohérence avec ces principes théoriques, la démarche de recherche en analyse interactionnelle se fonde sur une documentation de pratiques tutorales recueillies en contexte naturel et non provoqué, au moyen de traces audio-vidéographiques. Dans le cas présent, les enregistrements vidéo ont été effectués dans deux contextes professionnels à la fois distincts et présentant certaines similitudes. D’une part, le champ de la radiologie médicale en milieu hospitalier. D'autre part, celui de l'éducation de la petite enfance en contexte institutionnel.

Indépendamment des spécificités qui contrastent fortement selon les conditions d'exercice de ces deux métiers, il existe des convergences significatives entre les deux champs. Les formations des techniciens en radiologie médicale (TRM) et des éducateurs de l'enfance (EDE), même si elles ne relèvent pas du même niveau de reconnaissance dans le système éducatif suisse, sont proposées en alternance, combinant des périodes de stages et des périodes d'enseignement. L'expérience de travail en situation de stage fait l'objet d'un accompagnement par un praticien formateur ou un référent 
professionnel. Mais surtout, le travail des TRM tout comme celui des EDE porte sur des êtres humains (des enfants, des patients). Il implique l'instauration d'une relation interpersonnelle avec des " usagers ", dans une forme de prestation de service. C'est à ce titre que l'usage conjoint de ces deux situations retient notre attention.

\section{Encadré 1. Recueil et analyse des données audio-vidéo}

Les données empiriques exploitées dans le cadre de la présente recherche ont été recueillies entre 2012 et $2015(*)$, à l'occasion de stages effectués par des étudiants en formation professionnelle initiale, respectivement dans les domaines de la radiologie médicale et de l'éducation de l'enfance. Les stages font partie intégrante du parcours de formation. Ils alternent avec des périodes de formation en école professionnelle et visent la construction, chez les étudiants, d'une expérience pratique dans les conditions réelles de travail. À ce titre, les stages ont lieu dans des entreprises ou des institutions du monde du travail.

Dans chacun des deux contextes de travail, trois étudiants stagiaires ont été observés durant leur stage, durant trois demi-journées, et ce à différents moments de leur parcours de formation, soit en première et en troisième année. Les données totales disponibles s'élèvent ainsi à environ 25 heures d'enregistrements audio-vidéo de situations ordinaires de travail accompagné. Les situations enregistrées documentent des interactions entre les stagiaires, les tuteurs et les usagers des institutions concernées, respectivement des patients ou des enfants. Les enregistrements ont été réalisés à l'aide d'un dispositif vidéographique mobile, dans des situations ordinaires et non provoquées de travail, avec le consentement des participants. Les environnements de travail sélectionnés pour les enregistrements ont été retenus sur la base des critères suivants : a) selon les observations effectuées et le point de vue des participants, ils sont représentatifs des environnements professionnels proposés pour héberger des étudiants à l'occasion de stages, b) ils sont aménagés par des « praticiens formateurs » ou des « référents professionnels » qui endossent des fonctions tutorales à l'égard des stagiaires. Seules des institutions du secteur public ont donné leur accord pour prendre part à cette recherche.

Le traitement des données audio-vidéo suit un protocole en trois étapes, présenté de manière détaillée dans Filliettaz (2008). La première étape consiste en la confection de tableaux synoptiques de pré-analyse, dans lesquels les contenus des films sont décrits, structurés et hiérarchisés selon les phénomènes étudiés. La deuxième étape constitue une transcription détaillée et multimodale de séquences récurrentes du phénomène étudié (voir les conventions de transcription en Annexe de la version électronique de l'article). La troisième étape correspond à une analyse séquentielle de ces extraits retranscrits selon les catégories d'analyse présentées ci-dessous (positionnement, cadrage de l'activité, visibilité des savoirs).

${ }^{*}$ ) La recherche a été conduite avec le soutien du Fonds national suisse (FNS), sous les numéros de requête 100019_149759 et 100019_169743. 


\section{Les usagers, des ressources et des partenaires dans les interactions tutorales}

La démarche d'analyse empirique conduite ci-dessous porte sur deux extraits issus de chacun des deux corpus considérés. Les extraits se caractérisent par le fait qu'ils consistent tous deux en des situations de pratique professionnelle accompagnée, en présence des usagers. Les stagiaires y réalisent des activités de travail, adressées respectivement à des patients ou des enfants, en coprésence et avec le guidage de leur tutrice. L'analyse vise à décrire finement les formes de participation des usagers à ces interactions, l'influence de ces formes de participation sur les situations dans lesquelles s'engagent les stagiaires et les tutrices, et enfin leur effets sur les enjeux de formation qui président à ces situations.

Pour mener ces analyses, une attention particulière sera accordée à trois phénomènes interactionnels. À partir de travaux antérieurs, nous les considérons en effet comme des indicateurs significatifs des processus d'apprentissages en situation de travail (voir Filliettaz, Rémery \& Trébert, op. cit. ; Durand, Trébert \& Filliettaz, 2015).

Le premier axe d'analyse porte sur les formats de participation à l'interaction, qu'à la suite de Goffman, nous qualifions de "positionnement " (footing). Un des mérites des travaux de l'auteur $(1981 ; 1987)$ est d'avoir montré que les interactions verbales ne procèdent pas seulement d'une relation dyadique entre des " locuteurs " et des " récepteurs ", mais qu'elles consistent en des " rencontres sociales " dans lesquelles plusieurs participants peuvent se trouver engagés, selon des positions de participation diversifiées. Dans les situations de la vie quotidienne, les « récepteurs " d'un acte de communication peuvent, par exemple, être identifiés comme des destinataires ratifiés ou, au contraire, comme de simples témoins, perçus on non pas les participants à l'interaction. Les destinataires ratifiés peuvent également être désignés comme tels par le locuteur ou pas, selon que l'adressage est accompli de manière directe ou indirecte. Pour Goffman, ces processus de positionnement relèvent du " cadre participatif $»$ de l'interaction. En outre, ils présentent la caractéristique de se négocier et de se renégocier, tour de parole après tour de parole, par les participants eux-mêmes (Goodwin \& Goodwin, 2004). Dans le cadre des interactions tutorales, ces mécanismes ne sont pas sans enjeux dès lors qu'ils indiquent vers quels acteurs les participants (ré)orientent leur attention et dans quelle position de participation ils placent leurs partenaires à un moment donné de leur rencontre.

Un deuxième axe d'analyse des données porte sur les processus de " cadrage de l'activité " et des prémisses organisationnelles que les participants à l'interaction reconnaissent comme exerçant un rôle configurant dans la situation rencontrée. Toujours dans une perspective goffmanienne, les "cadres de l'expérience " (Goffman, 1991) permettent aux individus de répondre à une question qu'ils se posent, explicitement ou implicitement, à chaque instant de leur présence sur la scène constituée par la sphère publique : qu'est-ce qui se passe ici ? Ces cadres de l'expérience ne préexistent pas à leur rencontre et ne constituent pas des réifications tangibles et données une fois pour toutes. Ils sont rendus visibles à travers les 
comportements des participants et la signification que ces derniers leur conferent dans le processus intersubjectif de leur rencontre. " $\grave{A}$ partir du moment où nous comprenons ce qui se passe ", dit Goffman (Ibid., p. 242), "nous y conformons nos actions et nous pouvons constater en général que le cours des choses confirme cette conformité. Ce sont ces prémisses organisationnelles - que nous confirmons en même temps mentalement et par notre activité - que jappelle le cadre de l'activité ». C'est à ce titre que les cadres sont dits "vulnérables ». Ils peuvent être déconstruits et reconstruits au profit d'une autre interprétation de la situation. Et ils peuvent également être modalisés ou fabriqués dans des logiques d'hybridation, de transposition, voire de manipulation. C'est le cas, nous dit Goffman, des mises en scène dramaturgiques ou des "réitérations techniques" des apprenants, qui acquièrent certaines facultés par une répétition de gestes. Les phénomènes de cadrage de l'activité intéressent l'étude des situations d'apprentissage par le travail, dès lors que celles-ci peuvent servir d'indicateur de la manière dont les participants s'orientent vers des enjeux constructifs et/ou productifs à des moments particuliers de leur rencontre (Filliettaz \& Durand, op. cit.). En effet, il arrive parfois que l'activité de travail soit temporairement suspendue au profit de l'explicitation d'un savoir et de la conduite d'une activité de formation.

Enfin, un troisième axe d'analyse porte sur des enjeux épistémiques et sur la place des " savoirs » dans les interactions tutorales étudiées. Ces savoirs peuvent présenter des degrés d'explicitation et de visibilité variés selon les situations. Dans la perspective de la théorie de l'action conjointe en didactique comparée (Sensevy, Mercier \& Schubauer-Leoni, 2000), ces savoirs peuvent être transposés dans des milieux contrastés, selon des rythmes variés et à travers des formats de participation multiples. Dans les niveaux d'analyse microscopiques adoptés par certains courants en didactiques disciplinaires, ce sont bien les processus interactionnels qui permettent aux participants de thématiser les différentes "facettes" du savoir, rendues pertinentes dans la situation (Malkoun \& Tiberghien, 2008). Dans le contexte des interactions tutorales, ces questions ne sont pas sans enjeux dès lors qu'elles permettent de décrire selon quelles modalités des éléments de savoir en lien avec le travail sont à la fois mis en œuvre et partagés entre les participants, et comment ils peuvent devenir aussi, localement, des objets d'une pratique de transmission et de transposition (Filliettaz, 2009).

\subsection{Quand l'intervention de l'usager modifie la situation en radiologie médicale}

Le premier cas étudié relève du contexte de la santé. Il a été observé et enregistré dans le service de radiologie d'un centre hospitalier, au cours d'un stage de deuxième année, dans une salle de scanner. Le premier des deux extraits restitués ci-dessous porte sur l'accueil du patient et la préparation de son installation sur la " table " du scanner. La stagiaire (STA) explique au patient le déroulement de l'examen, en présence de sa tutrice (TUT). Le patient arrive en chaise roulante. Il est placé sous oxygène et doit 
subir une radiographie du thorax, qui implique l'injection d'un liquide de contraste au moment de réaliser l'examen par scanner.

\section{Vignette Extrait 1. L'accueil du patient au scanner}

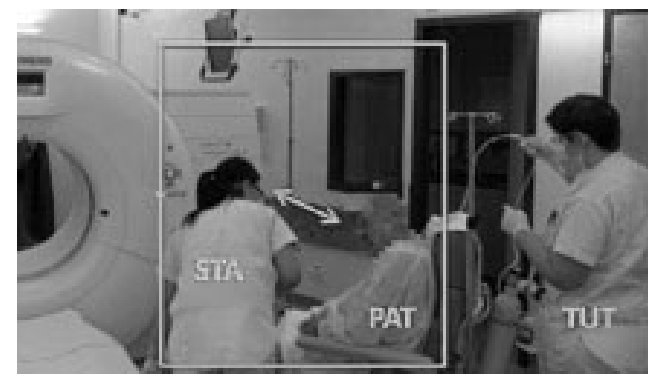

[\#1] STA (stagiaire) interagit avec le patient pendant que TUT (tutrice) mobilise le tuyau d'oxygène. Au cours de cette interaction, le patient demande s'il va devoir enlever le tuyau d'oxygène pendant l'examen.

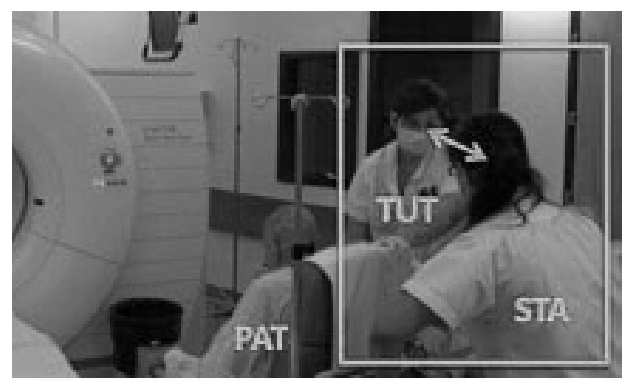

[\#2]TUT (tutrice) confirmeaupatient qu'il n'a pasbesoin d'enlever le tuyau d'oxygène. STA (stagiaire) interrompt son action sur la bouteille d'oxygène pour s'adresser à TUT (tutrice) et lui demande confirmation que l'examen peut être réalisé avec un apport d'oxygène au patient.

Source : (c) Equipe Interaction \& Formation.

Dans la vignette Extrait 1, dont la transcription détaillée est fournie en Annexe de la version électronique de l'article, les professionnelles confèrent au patient des positions variables dans l'interaction et investissent successivement la situation de différentes manières, qu'il importe de mettre en évidence.

Dans la partie inaugurale de l'extrait, et jusqu'à la ligne 13 (cf. Annexe électronique), un cadre de participation organisé en deux foyers d'action parallèles est instauré, dans lequel des activités complémentaires sont accomplies en vue de préparer le patient à l'examen. Dans un premier foyer, retranscrit dans la colonne de gauche, STA (stagiaire) conduit une interaction verbale avec PAT (patient), dans laquelle elle lui explique le déroulement prévu de l'examen (1. 1, 1. 2). Elle sélectionne ici PAT comme destinataire ratifié et désigné de ses propos, comme l'indiquent à la fois les choix pronominaux (vous), les contenus tropicaux, la direction de son regard et son orientation corporelle (voir [\#1]). De son côté, PAT ratifie ce cadre de participation et s'aligne dans la position de destinataire désigné dans laquelle il est placé. Il s'oriente vers STA, produit des signaux d'écoute (1. 3) et répond aux questions qui lui sont posées ("non", 1. 6 ; "J'ai la dialyse, mais c’est pas une allergie ", 1. 8). Sur le plan épistémique, des contenus de savoir sont mis en circulation dans l'interaction entre la stagiaire et le patient. Ils portent sur la procédure de l'examen médical et en détaillent différents sous-domaines : le positionnement du patient durant l'examen (1. 1) ; l'injection du liquide de contraste (1. 2) ; les contre-indications (1. 5). La mise en visibilité de ces contenus de savoir à destination du patient relève de la procédure de travail. Elle constitue 
un ensemble à la fois ordonné et routinisé de thèmes qui sont abordés au moment d'accueillir les patients dans ce contexte.

De son côté, la tutrice n'endosse clairement pas la même position interactionnelle que STA. À ce stade, elle n'interagit pas verbalement avec PAT, mais semble constituer un témoin perçu de son interaction avec STA. Elle ne reste cependant pas inactive pour autant. Elle s'engage dans une suite ordonnée d'actions non verbales, dont notamment la mobilisation du tuyau reliant le patient à la bombonne d'oxygène située à l'arrière de la chaise ([\#1]). Ces actions se déroulent en parallèle à l'interaction verbale menée par la stagiaire et le patient. Elles constituent donc un foyer d'action individuel accompli parallèlement à l'interaction STA-PAT et se synchronisant avec cette dernière. Les savoirs mobilisés par la tutrice dans le domaine de la préparation du patient ne sont pas rendus visibles sur le plan verbal, mais sont incorporés dans les manipulations réalisées.

L'intervention du patient en ligne 14 introduit des transformations significatives dans la situation et dans les conditions de participation des acteurs. En effet, à ce moment, le patient s'auto-sélectionne comme prochain locuteur et produit une demande de vérification à l'intention des professionnelles ("J'enlève le, l'oxygène alors ? ", 1. 14). Ce faisant, il place la stagiaire et la tutrice dans la position de destinataires désignés et induit un changement dans le cadre de participation. Ce n'est pas ici la stagiaire qui répond, mais la tutrice ("non non, on va le garder ", 1. 15). À son tour, la tutrice s'auto-sélectionne comme prochaine locutrice et catégorise le patient comme destinataire désigné.

C'est aussi à ce moment précis que la stagiaire interrompt l'activité non verbale dans laquelle elle était engagée, en manipulant la bouteille d'oxygène. Après avoir entendu la réponse de la tutrice au patient, elle s'auto-sélectionne comme prochaine locutrice et s'adresse de manière directe à la tutrice (" ah tu veux le laisser là? ", 1. 16). La tutrice ratifie ce nouveau cadre de participation en s'adressant à son tour à la stagiaire et en répondant à sa demande ("oui, normalement c'est assez long", 1. 17).

Sur le plan épistémique, l'intervention du patient induit également des modifications du rapport des acteurs à la situation. Alors que précédemment, le tuyau d'oxygène ne constituait, dans l'environnement, qu’un objet matériel manipulé par la tutrice, il devient, au moment de la question du patient, un thème de l'interaction verbale et donc un objet de l'activité, explicitement porté à l'attention de l'ensemble des participants. Ces derniers entretiennent des rapports asymétriques à cet objet de savoir. En effet, alors que la tutrice sait qu'il est possible de réaliser l'examen en maintenant un apport d'oxygène, la stagiaire et le patient semblent l'ignorer. Cette asymétrie n'est pas donnée a priori, mais induite par les formats de participation choisis par les participants, et en particulier par la manière dont ils endossent respectivement les positions de locuteur et de destinataire au moment où des enjeux épistémiques viennent structurer le cadre de l'activité. De ce point de vue, l'auto-attribution, par la tutrice, de son droit à la parole après la question formulée par le patient, agit comme une forme d'induction notable d'un rapport asymétrique au savoir (Heritage, 2012). 
À la suite de cette séquence de clarification concernant l'usage de l'oxygène durant l'examen, les tâches de préparation du patient se poursuivent par l'installation du patient sur la table du scanner. Cette installation ouvre de nouvelles formes de coopération entre les participants.

\section{Vignette Extrait 2 . L'installation du patient sur la table du scanner}

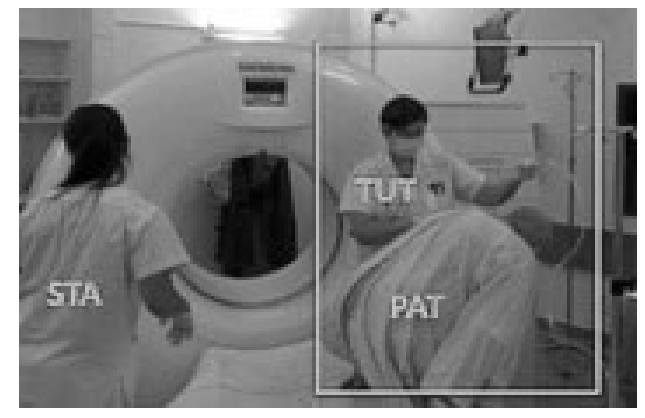

[\#1] TUT (tutrice) aide PAT (patient) à s'installer sur la table pendant que STA (stagiaire) se déplace sur la gauche du scanner.

Source : (c) Equipe Interaction \& Formation.

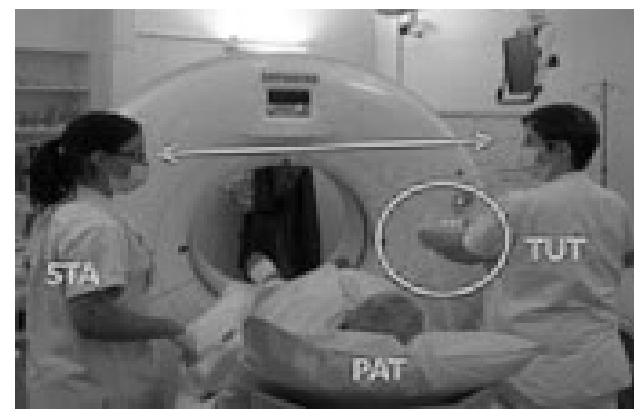

[\#2] TUT (tutrice) explique à STA (stagiaire) comment positionner le tuyau d'oxygène durant l'examen.

Dans ce deuxième extrait, des cadres de participation différents se mettent en place successivement, qui se stabilisent localement dans trois configurations distinctes et reconnaissables.

Dans un premier temps et jusqu'en ligne 31 (Cf. Annexe électronique), l'interaction s'organise selon une configuration similaire à celle qui avait prévalu au moment de l'accueil du patient, avec cependant une inversion des rôles entre la stagiaire et la tutrice. C'est ici la tutrice et non plus la stagiaire qui conduit l'interaction verbale avec le patient et qui le sélectionne comme destinataire désigné de ses propos ("voilà ", 1. 21 ; "je vais mettre le petit tuyau hop ", 1. 23 ; "allongez-vous ", 1. 26 ; "magnifique ", 1. 29). Les contenus verbalisés ponctuent l'accomplissement d'une suite ordonnée d'actions. Elles les commentent, les façonnent et les clôturent. Le patient s'aligne sur la position de participation dans laquelle il est placé par la tutrice. Il répond par des comportements non verbaux qui ratifient les demandes formulées. La stagiaire, pour sa part, participe au titre de témoin ratifiée à cette interaction avec le patient, et non plus au titre de locutrice. Comme la tutrice précédemment, elle s'engage dans des actions manipulatoires complémentaires qui se structurent dans un foyer d'action à la fois distinct et synchronisé avec la progression de l'installation du patient (voir [\#1]). Elle actionne ainsi le frein de la chaise, déplace le tuyau d'oxygène durant le déplacement du patient, installe le dispositif d'injection et actionne la commande de la table du scanner une fois que le patient est couché en position horizontale. 
Ensuite, en ligne 32, la tutrice opère des modifications significatives dans la configuration de participation, au moment où elle interpelle la stagiaire tout en manipulant le tuyau d'oxygène : "là tu sais tu le mets juste comme ça, tu vérifies qu'il y a la longueur, puis c'est bon hein". Ce faisant, elle sélectionne la stagiaire comme destinataire désignée, et place le patient dans la position d'un témoin perçu, mais auquel on ne s'adresse pas directement.

Sur le plan épistémique, cette séquence s'apparente à ce que Mayen \& Gagneur (2017, p. 10), à la suite de Deldalle (1967), désignent comme une "parenthèse intellective ". Elle permet de thématiser à nouveau l'objet de savoir que constitue le tuyau d'oxygène, mais dans un milieu qui présente des propriétés clairement didactiques. Cet objet de savoir est pointé à l'attention de la stagiaire. C'est là un bref moment de "transmission " ou de transposition, inséré, sous la forme d'un aparté, dans l'accomplissement d'une procédure de travail. La pertinence de cet objet de savoir s'ajuste localement aux conditions dans lesquelles il est mis en oeuvre et "montré ", mais la validité de ce savoir dépasse le contexte local et présente un caractère général. Cette parenthèse intellective n'est pas sans effets sur le cadre de l'activité tel qu'il est mis en place et rendu interprétable par les comportements des participants. Cette parenthèse "modalise " l'activité de travail dans un cadre d'un autre type, celui de l'apprentissage. Elle rend ainsi, à ce moment, particulièrement visibles les enjeux de formation qui président à la rencontre et les identités situées endossées par les participants sur le registre de la formation.

Mais cette parenthèse intellective ne constitue qu'une digression éphémère. Dès la ligne 34, la stagiaire met à nouveau en place une configuration de participation centrée sur une interaction verbale avec le patient, dans laquelle elle endosse la position de locutrice et le patient celle de destinataire désigné. C'est une séquence de consigne qui débute, dans laquelle la tutrice laisse la main à la stagiaire et endosse la position de témoin ratifié. À ce moment, les savoirs mis en circulation se centrent à nouveau sur la procédure d'examen et contribuent à accomplir le cadre de la procédure d'installation du patient. Le tuyau d'oxygène et ses conditions d'utilisation et de manipulation sortent alors du périmètre d'attention des participants.

Ce qui retient notre attention dans ce premier exemple, c'est d'abord la diversité des positions de participation dans lesquelles le patient peut être placé dans le cours du déroulement de cette interaction. Comme nous l'avons souligné, le patient peut être tantôt catégorisé comme un destinataire désigné auquel on s'adresse explicitement, tantôt comme un témoin perçu dont on parle, mais auquel on ne s'adresse pas (ex. 1. 16-17; 1. 32-33). Mais le patient endosse aussi parfois le rôle de locuteur et peut lui-même initier des échanges et placer les professionnelles dans des positions de destinataires. De ce point de vue, il ne fait pas que s'aligner sur les cadres de participation proposés par les soignants, il induit lui aussi des formats spécifiques de participation. 
Ces interventions à l'initiative du patient conduisent à des transformations notables de la situation, et ce sur différentes dimensions. Sur le plan de la participation, les interventions du patient "déplacent " de fait les autres participants. C'est le cas, notamment, lorsque la tutrice quitte son rôle de témoin pour répondre à la question du patient (1. 15). Et sur le plan épistémique, ses interventions ont le mérite de rendre saillants des éléments de l'activité jusqu'ici mis en œuvre, mais non thématisés. C'est le cas, notamment, du tuyau d'oxygène, dont la manipulation semble indiquer qu'une désinstallation est requise pour conduire l'examen. Or, c'est précisément la question du patient qui, séquentiellement, peut contribuer à déconstruire une inférence erronée, à partir de laquelle un savoir peut être mis en visibilité. Ainsi, il n'est pas nécessaire de désinstaller l'oxygénation d'un patient pour réaliser un examen par scanner du thorax. Il existe en effet des techniques de mobilisation et de positionnement du tuyau qui permettent d'éviter une telle désinstallation. C'est là tout l'enjeu d'une exploitation épistémique de l'étonnement du patient. Il s'agit d'en reconnaître la cause et de proposer des élaborations formatives sous la forme de "mises entre parenthèse " subreptices du travail. C'est du moins ce que révèle ici la propension des participants à s'orienter simultanément vers les objets du travail et vers les objets de savoir qui les sous-tendent.

\subsection{Des interactions parallèles, mais perméables : l'éducation de l'enfance}

Le second cas étudié relève du contexte de l'éducation de la petite enfance. Il a été observé et enregistré dans une institution de la petite enfance, au cours d'un stage de lère année.

Les extraits présentés et retranscrits ci-dessous restituent une situation typique relevant de la pratique professionnelle accompagnée. Une étudiante stagiaire (STA), en première année de formation, conduit une activité de psychomotricité avec un groupe de sept enfants âgés de 18 mois environ, en collaboration avec sa référente professionnelle (TUT). L'activité consiste à explorer librement différents objets, tels que des toboggans, des tapis, des cylindres ou des tunnels en mousse. L'activité a été conçue et préparée par la stagiaire, et validée par la référente à l'occasion d'un entretien pédagogique préparatoire.

La vignette présentée de l'extrait 3, retranscrite en Annexe de la version électronique de l'article, représente la seconde moitié du déroulement de cette activité. La stagiaire surveille l'activité de Tom, le plus jeune enfant présent, pendant que la référente joue avec un groupe d'enfants (Cal, Luc, Raf) qu' elle place dans un cylindre en mousse. 


\section{Vignette Extrait 3. Une activité de psychomotricité en contexte éducatif}

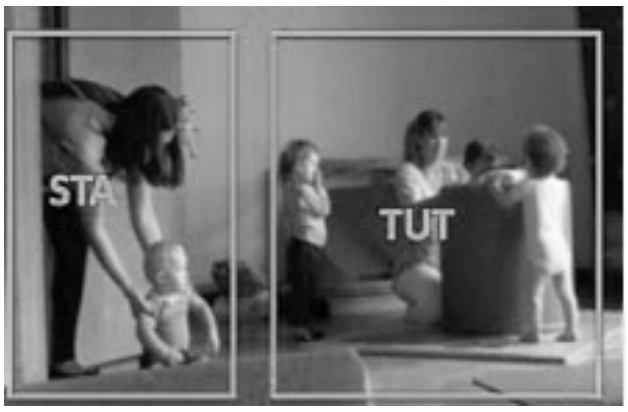

[\#1] STA ramène Tom dans la salle pendant que TUT (tutrice) joue avec un groupe d'enfants placés dans un cylindre en mousse.

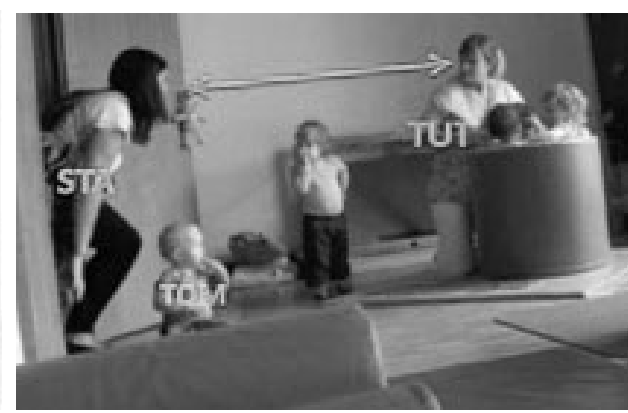

[\#2] STA (stagiaire) se lève et sollicite TUT (tutrice) du regard au moment de proposer à Tom de lui donner un ballon. Source : @ Equipe Interaction \& Formation.

La configuration de participation qui sous-tend l'interaction, au début de cet extrait, se structure clairement autour de deux foyers distincts (Goodwin \& Goodwin, op. cit. ; Markaki \& Rémery, 2016).

Un premier foyer interactionnel est pris en charge par la stagiaire et est orienté vers un enfant en particulier, Tom. La stagiaire sélectionne Tom comme destinataire direct de ses propos, le ramène dans l'espace d'activité ("Tom/ Tom/ tu restes là $\backslash$ tu restes ici ", l. 2) et lui propose un ballon pour maintenir son attention dans l'espace du jeu (" tu aimerais un ballon Tom/tu veux jouer avec un ballon ", 1. 7-8). La référente, pour sa part, s'engage dans un second foyer d'interaction, qu'elle partage avec un groupe de trois enfants placés dans un cylindre. Elle s'adresse d'abord au groupe d'enfants (" allez, vous faites la discothèque là ", 1.1 ; "est-ce quill y a une place pour Raf», 1. 5), puis à Raf en particulier ("viens, on essaie à trois/tu veux aller dedans ", 1. 6). L'espace de l'interaction est décomposé en "régions ", qui donnent lieu à des logiques d'organisation séquentielles spécifiques, et qui engagent des participants distincts (voir [\#1]). C'est là une configuration récurrente des situations de co-animation, dans lesquelles les éducatrices se distribuent des rôles complémentaires dans l'accomplissement du travail impliquant un groupe d'enfants (Filliettaz, Rémery \& Trébert, op. cit. ; Durand, Trébert \& Filliettaz, op. cit.).

Ces foyers d'interaction se déroulent certes de manière parallèle. Pour autant, ils ne sont pas étanches, mais entretiennent des relations d'interdépendance qui s'expriment de manière croissante dans le déroulement de l'activité. Pour commencer, observons que les « régions » qui composent l'espace de l'interaction ne sont pas cloisonnées et qu'elles rendent possibles des contacts visuels entre les participants. La stagiaire peut ainsi observer la conduite de l'activité de la référente. Réciproquement, la référente peut maintenir un contact visuel avec l'activité de la stagiaire. C'est d'ailleurs ce qui se passe au moment où la stagiaire formule, à l'attention de Tom, l'offre de lui donner un ballon (1. 8-9). Au moment de se lever et 
de formuler son offre ("tu veux jouer avec un ballon/peut-être »), elle oriente son regard en direction de la référente. Celle-ci s'aligne à son regard tout en terminant de placer Raf dans le cylindre (voir [\#2]). Elle s'engage donc ici simultanément dans deux foyers, dans lesquels elle endosse des rôles distincts.

Cette perméabilité observée entre les deux foyers de l'activité éducative se confirme et se renforce dans la suite de l'interaction, au moment notamment où Cal, un des trois enfants enfermés dans le cylindre bercé par la tutrice, porte son attention vers les ballons que la stagiaire distribue à Tom.

\section{Vignette Extrait 4. La distribution des ballons aux enfants}

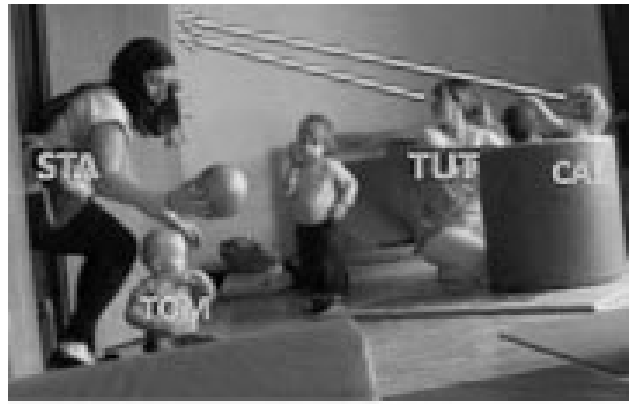

[\#1] Au moment où STA (stagiaire) tend le ballon à Tom, Cal et TUT (tutrice) regardent en direction de l'armoire où sont rangés les ballons.

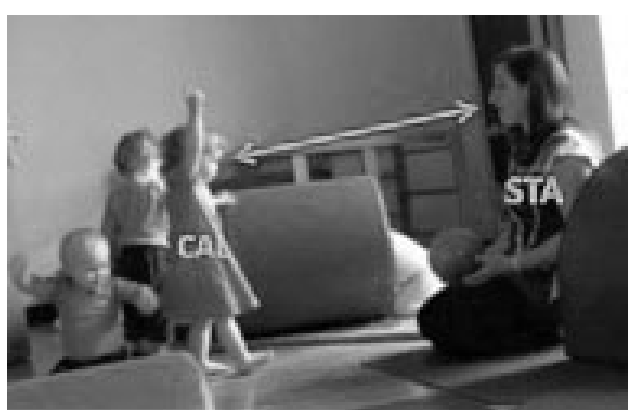

[\#2] Cal s'approche de STA (stagiaire) en lui demandant un ballon.

Source : @ Equipe Interaction \& Formation.

Ainsi, en ligne 11 émerge une nouvelle forme de perméabilité entre les foyers d'interaction précédemment établis. Au moment de voir la stagiaire sortir un ballon de l'armoire pour le tendre à Tom, Cal, une petite fille « prisonnière » du cylindre, oriente son regard et tend la main en direction de l'armoire, manifestant son intérêt pour les ballons ("é-ba-é-li-li », 1. 11). De manière intéressante, la référente s'aligne visuellement à cette sollicitation (voir [\#1]) et lui attribue une signification ("les ballons?», 1. 12). Elle répond ensuite à la sollicitation de Cal en désignant explicitement la stagiaire comme la personne de référence pour la conduite de l'activité (" ah je sais pas; je sais pas si les ballons vont être dans notre activité hein, c'est STA qui mène ", 1. 13). Comme l'indique la transcription, la dernière partie de la réponse de la référente est formulée, sur le plan prosodique, au moyen d'une augmentation du volume de la voix. Ce faisant, elle inclut également les autres participants, dont la stagiaire, comme des destinataires ratifiés de son propos. Elle accomplit ici ce que KerbratOrecchioni (1992) décrit comme un "trope communicationnel»" s'adressant indirectement à la stagiaire, par l'intermédiaire d'une désignation directe de l'enfant comme allocutaire.

4. un énoncé adressé à quelqu’un peut contenir en fait un message indirectement adressé à une autre per- 
La suite de l'interaction se déploie à nouveau sur le registre de deux foyers d'action conduits parallèlement. La tutrice continue de bercer les enfants dans le cylindre, selon la métaphore du bateau (1. 13), pendant que la stagiaire fait rouler le ballon en direction de Tom (1. 15), qui le lui renvoie (1. 16). Au terme du basculement du cylindre, Cal se relève et s'approche de la stagiaire, occupée à faire rouler le ballon en direction de Tom (1.21). Elle s'engage ici plus fortement encore dans le foyer d'activité placé sous la responsabilité de la stagiaire (voir [\#2]). Cette dernière valide cette sollicitation implicite (" taimerais un ballon ?", 1. 22), tend le ballon à Cal ("alors celui-là, tu peux le donner à Tom et je vais t'en prêter un autre", 1. 24) et se dirige vers l'armoire pour distribuer des ballons aux autres enfants présents dans la salle. Dès ce moment, l'ensemble des enfants présents délaissent progressivement le cylindre pour se regrouper autour de la stagiaire.

Du point de vue de l'accomplissement collectif d'une activité éducative, cette étude de cas permet de souligner la grande plasticité des configurations de participation et le travail permanent de (re)contextualisation engagé par les éducatrices. Comme indiqué par le déroulement séquentiel de cette interaction, les modalités à travers lesquelles les participants s'orientent dans les activités se transforment en permanence, de nouveaux foyers d'interaction pouvant émerger, en contaminer d'autres, pour finir par les intégrer complètement.

Mais surtout, ces extraits permettent également de montrer comment la référente, tout en conduisant avec les enfants des activités éducatives, maintient un engagement en direction de la stagiaire et rend manifeste son orientation vers des enjeux de formation. Par exemple, en accomplissant une activité de bercement des enfants de manière ostensive, et par une prosodie marquée comme publiquement adressée, elle porte à l'attention de la stagiaire qui l'observe, des usages possibles du matériel de jeu (Filliettaz, Rémery \& Trébert, op. cit.). Par ailleurs, en s'alignant sur une sollicitation visuelle de la part de la stagiaire, produite au moment précis de proposer le jeu du ballon à Tom, la référente valide implicitement l'offre et le choix effectués par la stagiaire d'introduire un nouvel objet dans le jeu. Enfin, en adressant " à la cantonade " qu'elle "ne sait pas si les ballons vont être dans notre activité " et que "c'est STA qui mène ", elle place la stagiaire dans une position de destinataire indirecte de son propos. Elle porte aussi ostensiblement à son attention le fait que d'autres enfants souhaitent également jouer avec les ballons. Autrement dit, même sans s'adresser de manière directe et explicite à la stagiaire, la référente agit sur ses conditions de participation, a) en légitimant sa position d'éducatrice en charge de la conduite de l'activité, b) en validant ses choix relatifs à la progression de l'activité, c) en portant à son attention la pertinence d'envisager une relance de l'activité pour l'ensemble des enfants présents. Ainsi, la référente produit une forme de guidage de l'activité qui trouve d'autres voies d'expression que celles de consignes explicites, mais qui recourt à un large éventail de ressources multimodales, dont les modulations du volume de la voix, les orientations visuelles et les formes indirectes d'adressage. C'est par la mise en ouvre de ces ressources interactionnelles qu' elle rend

sonne témoin de la discussion. 
manifeste son orientation dans un double contexte, celui d'une activité éducative ciblée sur les enfants, et celui d'un guidage tutoral ciblé sur la stagiaire.

Notons que les enfants ne sont pas étrangers aux processus de guidage à l'œuvre dans cette situation. En particulier, les formes d'engagement de Cal contribuent activement à la reconfiguration progressive des cadres de participation et de la distribution des tâches et des rôles entre la tutrice et la stagiaire. En portant progressivement son attention vers les ballons (1. 11, 1. 21, 1. 23), Cal sollicite de manière croissante la stagiaire et marque un intérêt progressif et contagieux pour l'activité placée sous sa responsabilité. Dans ce processus, la stagiaire, avec l'aide de Cal, reprend pour ainsi dire la main sur une activité de psychomotricité qu'elle est supposée animer et dont elle était devenue en partie périphérique.

Par ailleurs, en s'interessant progressivement aux ballons, Cal contribue à thématiser un objet significatif de la professionnalité des éducatrices, soit leur capacité à " relancer » des activités en introduisant progressivement des objets nouveaux dans l'environnement qui permettent de susciter l'intérêt des enfants pour l'activité. L'usage du ballon comme médiation d'une relance de l'activité psychomotrice constitue ici un indice de structuration de l'activité éducative et un savoir-faire qu'il s'agit aussi d'apprendre et de faire apprendre. De ce point de vue, conférer de la visibilité et de la légitimité à l'intérêt manifesté par Cal aux ballons qu'elle aperçoit n'est pas sans effets sur le potentiel d'apprentissage de la situation.

Cela confere de fait un statut épistémique à un dilemme de structuration de l'activité éducative. En effet, en déclarant de manière ostensible, à l'attention de Cal, qu'elle a bien repéré son intérêt pour les ballons, mais que ce n'est pas elle "qui gère » (1. 12), la tutrice invite à son tour la stagiaire à accorder son attention à cet intérêt et à considérer la possibilité de collectiviser la relance auprès des autres enfants et non plus seulement auprès de Tom. La suite de l'activité confirme que c'est bien vers cette généralisation que la stagiaire s'oriente à son tour. Elle semble donc avoir bien repéré, dans l'environnement, cet indice implicite et indirect placé par la référente sur le comportement des enfants.

\section{Conclusion : quelles implications pour la formation ?}

Les deux études de cas présentées dans cet article illustrent le fait que les usagers des interactions de services ne sont pas des agents inertes ou de simples « objets » du travail. Ils doivent au contraire être reconnus comme des acteurs à part entière, qui contribuent à faire évoluer localement les situations de travail et qui agissent sur les conditions de participation des stagiaires et des tutrices, dans une logique d'interdépendance. C'est ce que révèle, dans le cas des examens en radiologie médicale, les formes auto-initiées des interventions des patients et, dans le cas des activités éducatives, l'orientation des enfants dans les différents foyers d'activité qui leur sont proposés. Comme nous l'avons vu, la présence et les formes de participation des usagers conduisent fréquemment à des formes complexes et stratifiées de rencontres sociales, qui peuvent se structurer autour d'une pluralité de foyers d'interactions, accomplis de manière parallèle et symétrique. Elles rendent possibles des phénomènes de scission de l'interaction et des formes plus ou moins stabilisées de multi-activités. C'est là 
un des effets sur la participation des contingences spécifiques liées aux activités de service impliquant des êtres humains.

Les formes d'intervention des usagers dans les situations de travail ne sont pas sans conséquences sur le potentiel d'apprentissage et donc sur les enjeux de formation qui président aux interactions de tutelle. Selon les observations effectuées dans les données considérées ici, trois types d'effets majeurs s'observent.

En premier lieu, il apparaît que les interventions des usagers dans les formats de participation à l'interaction agissent sur le statut des objets matériels et leur signification en acte. Par exemple, c'est ce que montre la question du patient à propos du "tuyau d'oxygène ", en plaçant un objet technique au centre d'une attention partagée entre les participants. Et c'est également ce que révèle l'intérêt progressif des enfants pour le "ballon ", qui va conduire à une réorganisation notable de la configuration de la participation à l'interaction.

Deuxièmement, et en prolongement du point précédent, les interventions des usagers dans les situations contribuent à conférer de la visibilité à des contenus épistémiques jusqu'ici tacites, incorporés, et donc non manifestes pour l'ensemble des participants. Le " tuyau d'oxygène " devient une catégorie de savoir nécessaire à la conduite de l'examen radiologique ; le «ballon » devient un instrument permettant de relancer une activité psychomotrice.

Enfin, les formes actives de participation des usagers créent des opportunités d'étayage de l'activité des stagiaires et ainsi des occasions de mise en visibilité de l'activité des tuteurs sur le registre de l'accompagnement en situation de travail. La question du patient donne lieu à des " parenthèses intellectives " et à de brèves séquences de transmission de contenus à destination de la stagiaire. Et l'orientation des enfants vers les ballons conduit à une confirmation implicite d'un moment judicieux pour relancer l'activité psychomotrice.

De manière intéressante, il apparaît aussi, dans nos exemples, que les exploitations formatives des transformations induites par les usagers ne constituent pas, en tant que telles, des opportunités d'apprentissage, mais qu'elles résultent au contraire d'un accomplissement lui-même réalisé collectivement par les participants. Autrement dit, elles sont des accomplissements interactionnels, vers lesquels les participants doivent s'orienter. C'est ce que l'on observe, dans le cas de l'accueil du patient en radiologie, lorsque la tutrice et la stagiaire explicitent et élaborent les contenus de savoir associés à l'usage du tuyau d'oxygène ou, dans le cas de l'éducation de l'enfance, lorsque la stagiaire repère bien les indices de guidage proposés par la tutrice en direction de la relance de son activité. Dans chacun de ces deux exemples, les interventions des usagers sont détournées de leur fonction instrumentale première pour guider l'activité des stagiaires. Elles procèdent, pourrait-on dire, d'une " genèse instrumentale ", au sens de Rabardel (1995, p. 123), c'est-à-dire d'un "écart entre le prévu et le réel dans l'utilisation des artefacts". Le tuyau d'oxygène devient le support d'une brève formation à son utilisation en situation de radiographie par scanner ; la référence au ballon, une invitation à repérer des opportunités de relance dans une activité éducative. Comme nous l'avons vu, ces genèses instrumentales peuvent être plus ou moins explicites ou implicites, directes ou indirectes. Elles peuvent prendre des formes plus ou moins visibles pour 
les participants et être plus ou moins facilement reconnues comme telles par eux. Tout l'enjeu est donc de les repérer et d'en faire des objets interprétables et potentiellement apprenants par les participants.

Dès lors, quelles sont les conséquences de ces observations sur la formation professionnelle ? Dans un contexte au sein duquel les activités de service sont appelées à se développer, il importe avant tout de repenser la place des usagers, non seulement dans les activités de travail, mais aussi dans les enjeux de formation à ces pratiques.

Comme nous l'avons vu ici, les usagers ne sont pas que les « destinataires » du travail ou des " objets " sur lesquels il porte. Ce sont aussi des " ressources » pour apprendre le travail et des partenaires qui agissent sur les conditions dans lesquelles le travail s'apprend. Par conséquent, les programmes de formation aux métiers de service ne peuvent pas se contenter de construire des connaissances sur les destinataires du travail (ex. l'anatomie du patient ou le développement cognitif et socio-affectif de l'enfant). Ils doivent également s'appliquer à mieux comprendre comment ces destinataires peuvent contribuer activement à la formation et reconnaître la place et le rôle qu'ils endossent dans les pratiques de formation en situation de travail.

Plus généralement, la démarche adoptée ici invite à un regard renouvelé sur les interactions tutorales et les pratiques de transmission du travail. À la lumière des données recueillies et analysées dans cet article, les pratiques de transmission ne se conçoivent plus seulement comme un processus bidirectionnel et binaire impliquant des « experts » et des " novices". Ce sont au contraire des polylogues accomplis sous la forme de cadres de participation complexes. Les participants peuvent s'y s'engager successivement, alternativement ou simultanément, dans une pluralité de foyers d'attention et d'action, et les " usagers ", même s'ils ne disposent pas d'une expertise avérée ou reconnue, contribuent à la mise en visibilité de contenus épistémiques. À l'heure où la didactique professionnelle se redéfinit comme "l'analyse du travail pour rendre le travail formateur " (Mayen \& Gagneur, op. cit., p. 72) et où elle cherche à repenser la formation en situation de travail autrement que comme un apprentissage sur le tas, ce changement de regard sur les " usagers " du travail semble particulièrement pertinent. Il illustre les méthodes par lesquelles les acteurs du travail et de la formation peuvent agir sur les situations pour en soutenir et en développer le potentiel d'apprentissage. C'est là, pensons-nous, que réside l'intérêt du travail analytique fin qui permet de rendre ces transformations visibles.

\section{Bibliographie}

Balslev K., Filliettaz L., Ciavaldini-Cartaut S. \& Vinatier I. (Ed.) (2015), La part du langage : pratiques professionnelles en formation, Paris, L'Harmattan.

Billett S. (2001), Learning in the Workplace. Strategies for effective practice, Crows Nest, Allen \& Unwin. 
Boccara V., Vidal-Gomel C., Rogalski J. \& Delhomme P. (2015), "A longitudinal study of driving instructor guidance from an activity-oriented perspective", Applied ergonomics, 46, pp. 21-29.

Borzeix A. \& Fraenkel B. (Ed.) (2001), Langage et travail. Communication, cognition, action, Paris, CNRS.

Boutet J. (2001), "La part langagière du travail : bilan et évolution ", Langage et Société, 98, pp. 17-42.

Boutet J. (2008), La vie verbale au travail, Toulouse, Octarès.

Cerf M. \& Falzon P. (2005), Situations de service : travailler dans l'interaction, Paris, Presses universitaires de France.

Chrétien F. (2014), "La relation de tutorat dans les espaces-test agricoles : une mise à l'épreuve de l'apprendre et du transmettre », Travail et Apprentissages, 13, pp. 51-72.

Deldalle G. (1967), Lidée d'expérience dans la philosophie de John Dewey, Paris, Presses universitaires de France.

Durand I., Trébert D. \& Filliettaz L. (2015), « Offre et prise de place : l'accomplissement des configurations de participation à l'interaction tutorale ", in Balslev K., Filliettaz L., Ciavaldini-Cartaut S. \& Vinatier I. (Ed.), La part du langage : pratiques professionnelles en formation, Paris, L'Harmattan, pp. 31-60.

Filliettaz L. (2008), "L'analyse des interactions ", in Filliettaz L., de Saint-Georges \& Duc B., Vos mains sont intelligentes! : Interactions en formation professionnelle initiale, Université de Genève : Cahiers de la Section des Sciences de l'Éducation, 117, pp. 71-114.

Filliettaz L. (2009), " Les formes de didactisation des instruments de travail en formation professionnelle initiale », Travail et Apprentissages, 4, pp. 26-56.

Filliettaz L. (2010), "Guidance as an interactional accomplishment : Practice-based learning within the Swiss VET system”, in Billett S. (Ed.), Learning through practice : Models, traditions, orientations and approaches, Dordrecht, Springer, pp. 156-179.

Filliettaz L. (2014), "L'interaction langagière : un objet et une méthode d'analyse en formation d'adultes ", in Friedrich J. \& Pita J. (Ed.), Recherches en formation des adultes : un dialogue entre concepts et réalité, Dijon, Editions Raisons et Passions, pp. 127-162.

Filliettaz L. (2015), « Les ressources interactionnelles de la structuration des activités en contexte éducatif ", Bulletin suisse de linguistique appliquée, 101, pp. 11-26.

Filliettaz L., de Saint-Georges I. \& Duc B. (2008), “Vos mains sont intelligentes! " : Interactions en formation professionnelle initiale ", Université de Genève : Cahiers de la Section des Sciences de l'Education, 117. 
Filliettaz L. \& Durand I. (2016), "L'activité de conception des tuteurs comme modalisation de l'expérience du travail : le cas de la formation des techniciens en radiologie médicale ", Sciences de l'éducation pour l'Ère nouvelle, 49, pp. 83-109.

Filliettaz L. \& Rémery V. (2015), «Transmettre le travail par les mises en formes langagières de l'activité ", in Wittorski R. (Ed.), La transmission du travail, Nîmes, Champ social, pp. 47-81.

Filliettaz L., Rémery V. \& Trébert D. (2014), « Relation tutorale et configurations de participation à l'interaction. Analyse de l'accompagnement des stagiaires dans le champ de la petite enfance », @ctivités, 11(1),pp. 22-46.

Filliettaz L. \& Trébert D. (2016), «Coanalyse des interactions tutorales et communication en contexte de formation professionnelle des éducatrices de l'enfance : Regards croisés sur la fonction de référente professionnelle ", Communiquer, 18, pp. 85-102.

Fuller A., \& Unwin L. (2003), "Learning as apprentices in the contemporary UK workplace: creating and managing expansive and restrictive participation", Journal of Education and Work, 16(4), pp. 407-426.

Garfinkel A. (2007), Recherches en ethnométhodologie, Paris, Presses universitaires de France.

Goffman E. (1981), Footing. In Forms of Talk, Philadelphia: University of Philadelphia Press, pp. 124-159.

Goffman E. (1987), "La position ", in Façons de parler, Paris, Editions de Minuit, pp. 133-166.

Goffman E. (1991), Les cadres de l'expérience, Paris, Editions de Minuit.

Goodwin C. (2000), "Action and embodiment within situated human interaction”, Journal of Pragmatics, 32, pp. 1489-1522.

Goodwin C. \& Goodwin M.H. (2004), "Participation”, in Duranti A. (Ed.), A Companion to Linguistic Anthropology, Oxford: Basil Blackwell, pp. 222-244.

Heritage J. (2012), "Epistemics in action: Action formation and territories of knowledge", Research on Language \& Social Interaction, 45(1), pp. 1-29.

Hubault F. (Ed.) (2002), La relation de service, opportunités et questions nouvelles pour l'ergonomie, Toulouse, Octarès.

Joseph I. (1995), "Le temps partagé : le travail du machiniste-receveur ", in Joseph I. \& Jeannot G. (Ed.), Métiers du public. Les compétences de l'agent et l'espace de l'usager, Paris, CNRS, pp. 63-83.

Kerbrat-Orecchioni C. (1992), Les interactions verbales, vol. 2, Paris, Armand Colin.

Kress G. et al. (2001), Multimodal teaching and learning. The rhetorics of the science classroom, London: Continuum. 
Kunégel P. (2011), Les maîtres d'apprentissage. Analyse des pratiques tutorales en situation de travail, Paris, L'Harmattan.

Malkoun L. \& Tiberghien A. (2008), «Objets de savoir et processus scientifiques en jeu dans les productions discursives en classe de physique de lycée ", in Filliettaz L. \& Schubauer-Leoni M.-L. (Ed.), Processus interactionnels et situations éducatives, Bruxelles, De Boeck.

Markaki V. \& Rémery V. (2016), «Documenter l'activité tutorale en situation de travail : pour une approche du travail en actes ", Sociologies et société, XLVIII, n 1, pp. 143-167.

Mayen P. (2002), "Le rôle des autres dans le développement de l'expérience ", Education Permanente, 151, pp. 87-107.

Mayen P. \& Gagneur Ch.-A. (2017), « Le potentiel d'apprentissage des situations : une perspective pour la conception de formations en situations de travail ", Recherches en Education, 28, pp. 70-83.

Mikkonen S. et al. (2017), "Guiding workplace learning in vocational education and training: a literature review”, Empirical Research in Vocational Education and Training, 9(9).

Mondada L. (2004), «Temporalité, séquentialité et multimodalité au fondement de l'organisation de l'interaction : le pointage comme pratique de prise de tour ", Cahiers de linguistique française, 26, pp. 269-292.

Pastré P. (2011), La didactique professionnelle, Paris, Presses universitaires de France.

Pastré P., Mayen P. \& Vergnaud G. (2006), «La didactique professionnelle », Revue française de pédagogie, 154, pp. 145-198.

Rabardel P. (1995), Les hommes et les technologies. Approche cognitive des instruments contemporains, Paris, Armand Colin.

Rémery V. \& Markaki V. (2016), "Travailler et former : l'activité hybride des tuteurs ", Education Permanente, 206, pp. 47-59.

Sacks H. (1992), Lectures on conversation, 2 volumes, Oxford : Blackwell.

Savoyant A. (1995), "Guidage de l'activité et développement des compétences dans une entreprise d'insertion ", Education Permanente, 123, pp. 91-99.

Schegloff E. (2007), Sequence organization in interaction: A primer in conversation analysis, Cambridge: Cambridge University Press.

Sensevy G., Mercier A. \& Schubauer-Leoni M.-L. (2000), "Vers un modèle de l'action didactique du professeur ", Recherches en didactique des mathématiques, 20(3), pp. 263-304.

Traverso V. (2016), Décrire le français parlé en interaction, Paris, Ophrys. 